Balzer, K. M.; Witte, Hartmut; Faustmann, P.; Kozianka, J.; Waleczek, $H$.:

Die vaskuläre Anatomie der vorderen Bauchwand : ein Beitrag zur Vermeidung von Gefäßverletzungen bei der laparoskopischen Chirurgie

URN: $\quad$ urn:nbn:de:gbv:ilm1-2014210072

Published OpenAccess: September 2014

Original veröffentlicht in:

Chirurgische Gastroenterologie : mit interdisziplinären Gesprächen. - Basel :

Karger (ISSN 1421-9808). - 19 (2003) 1, S. 81-85.

DOI: $\quad 10.1159 / 000070521$

URL: $\quad$ http://dx.doi.org/10.1159/000070521

[Visited: 2014-08-29]

„Im Rahmen der hochschulweiten Open-Access-Strategie für die Zweitveröffentlichung identifiziert durch die Universitätsbibliothek IImenau."

"Within the academic Open Access Strategy identified for deposition by IImenau University Library."

„Dieser Beitrag ist mit Zustimmung des Rechteinhabers aufgrund einer (DFG-geförderten) Allianz- bzw. Nationallizenz frei zugänglich."

"This publication is with permission of the rights owner freely accessible due to an Alliance licence and a national licence (funded by the DFG, German Research Foundation) respectively."

\section{DFG}

Nationallizenzen 


\title{
Die vaskuläre Anatomie der vorderen Bauchwand: Ein Beitrag zur Vermeidung von Gefäßverletzungen bei der laparoskopischen Chirurgie*
}

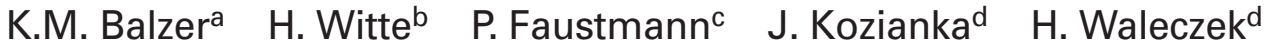 \\ ${ }^{a}$ Klinik für Gefäßchirurgie und Nierentransplantation, Heinrich-Heine-Universität Düsseldorf \\ ${ }^{b}$ Institut für Spezielle Zoologie und Evolutionsbiologie, Friedrich-Schiller-Universität Jena \\ ${ }^{c}$ Abteilung für Neuroanatomie und Molekulare Hirnforschung, Ruhr-Universität Bochum \\ ${ }^{d}$ Chirurgische Klinik, St. Anna Hospital Herne, Deutschland
}

\section{Schlüsselwörter}

Laparoskopische Chirurgie · Gefäßverletzungen

\section{Zusammenfassung}

Die Verletzung der in der Bauchwand verlaufenden Gefäße ist eine potentielle Komplikation aller laparoskopischer Verfahren. Die Kenntnis der Anatomie bildet die Grundvoraussetzung zur Vermeidung derartiger Komplikationen. An 28 Leichnamen wurde der Verlauf der Gefäße der Bauchwand bestimmt und Regionen für eine sichere sowie solche für eine gefährliche Trokarposition (dangerous zones) aufgezeigt.

\section{Einleitung}

Auf dem Weg, die Sicherheit laparoskopischer Eingriffe weiter zu verbessern, will die vorliegende Untersuchung die Aufmerksamkeit auf die vaskuläre Bauchwand-Anatomie lenken,

\footnotetext{
**Die vorliegende Arbeit besteht zu Teilen aus Ergebnissen der Dissertation von Herrn Dr. med. Kai M. Balzer an der Medizinischen Fakultät der Ruhr-Universität Bochum.
}

\author{
Key Words \\ Laparoscopic surgery · Vascular injury
}

\section{Summary}

Injuries to vessels of the abdominal wall are an potential complication in laparoscopic surgery but, with the increasing use of this technique, tend to occur more often. The exact knowledge of the anatomy of the parietal structures of the abdominal wall remains the main prerequisite in avoiding these complications. In this study, the course of these vessels was dissected in 28 abdominal walls taken from human cadavers, and regions for relatively safe trocar positions and 'dangerous zones' were determined. deren Kenntnis die Grundlage zur Vermeidung von Gefäßverletzungen bei der Trokarpositionierung darstellt. Verletzung der Bauchwand-Gefäße haben potentiell negative Folgen für Patienten, so z.B. Infektion des Hämatoms oder Laparotomie bei persistierender Blutung [1-4].

Milloy et al. [5] untersuchten den Verlauf des Musculus rectus abdominis und der Arteria epigastrica in Bezug auf die Gefäßversorgung und der Häufigkeit von Anastomosen schon 1960, in Bezug auf eine genaue Lokalisation bzw. die Vermeidung einer Verletzung derselben werden in der anatomischen

\begin{tabular}{ll}
\hline KARGER & ๑ 2003 S. Karger GmbH, Freiburg \\
Fax +49 7614520714 & Accessible online at: \\
$\begin{array}{l}\text { E-mail Information@Karger.de } \\
\text { www.karger.com }\end{array}$ & www.karger.com/cga
\end{tabular}


Literatur aber nur ungenaue Angaben gemacht: Die A. epigastrica verläuft innerhalb der Rektusscheide hinter dem M. rectus abdominis, 2 Querfinger lateral vom Nabel schräg aufwärts [6].

In älteren Studien zu Komplikationen bei laparoskopischen Eingriffen werden diese Verletzungen mit Häufigkeiten von 0,015-0,5\% angegeben [7-9]. Neuere Untersuchungen geben jedoch Häufigkeiten von $0,8 \%$ bei der laparoskopischen Hernienchirurgie, 3\% bei der laparoskopischen Kolonchirurgie und 3,4\% bei der laparoskopischen Refluxchirurgie an [10]. Nur 4 von 110 Autoren machen jedoch zu trokarassoziierten Blutungen der Bauchwand überhaupt Angaben. Obwohl sie in einigen Publikationen durchaus in relevanter Häufigkeit auftraten, findet dieses Problem bislang offensichtlich noch wenig Beachtung.

Zur Vermeidung von Blutungen aus Bauchwand-Gefäßen ist die routinemäßige präoperative Duplexsonographie der Bauchwand beschrieben worden [11], die jedoch wegen des damit verbundenen Zeitaufwands auf Einzelfälle beschränkt bleiben wird. Die üblicherweise vor einer Trokarplatzierung angewendete Transillumination [12] kann nach Quint et al. [13] die tiefen, in der Bauchwand verlaufenden Gefäße häufig nicht ausreichend sicher identifizieren. Die Verwendung von stumpfen anstelle von scharfen Trokarspitzen konnte das Risiko einer Gefäßverletzung in der Bauchwand deutlich reduzieren [10, 14]. Die Kenntnis von «dangerous zones» bleibt aber eine Grundvoraussetzung zur Vermeidung einer solchen Verletzung. Sie stellt die für Patienten günstigste Verfahrensweise dar.

\section{Material und Methoden}

Aus 28 formalinfixierten Leichnamen, 10 männlichen und 18 weiblichen wurden Thorax- und Bauchwand entnommen und die Gefäßverläufe (Arteriae epigastricae superior, inferior, Ramus ascendens der Arteria circumflexa ilium profunda) sowie der Verlauf des M. rectus abdominis anhand eines Koordinatensystems morphometrisch bestimmt (Abb. 1) und Mittelwert, Standardabweichung, der Median und das 95\%-Konfidenzintervall errechnet. Die Messungen erfolgten für die A. epigastrica bezogen auf die Medianlinie (Processus xiphoideus - Symphyse) und für den R. ascendens der A. circumflexa ileum profunda bezogen auf den lateralen Rand des M. rectus abdominis. Der Verlauf der Aa. epigastricae inferior und superior wurde mit dem Verlauf der Gegenseite und dem Verlauf des M. rectus abdominis mittels Pearson's Korrelationstest verglichen.

\section{Ergebnisse}

Auf Höhe der Symphyse konnte die A. epigastrica inferior links mit einer mittleren Entfernung zur Mittellinie von 6,38 $\pm 0,88 \mathrm{~cm}$ (Mittelwert \pm Standardabweichung) bei einem $95 \%$ Konfidenzintervall von 5,97-6,79 cm dargestellt werden. Rechts betrug der Mittelwert 6,59 $\pm 0,83 \mathrm{~cm}$ bei einem 95\%Konfidenzintervall von 6,18-7,00 cm. Zwischen männlichen und weiblichen Präparaten ergaben sich keine Unterschiede.

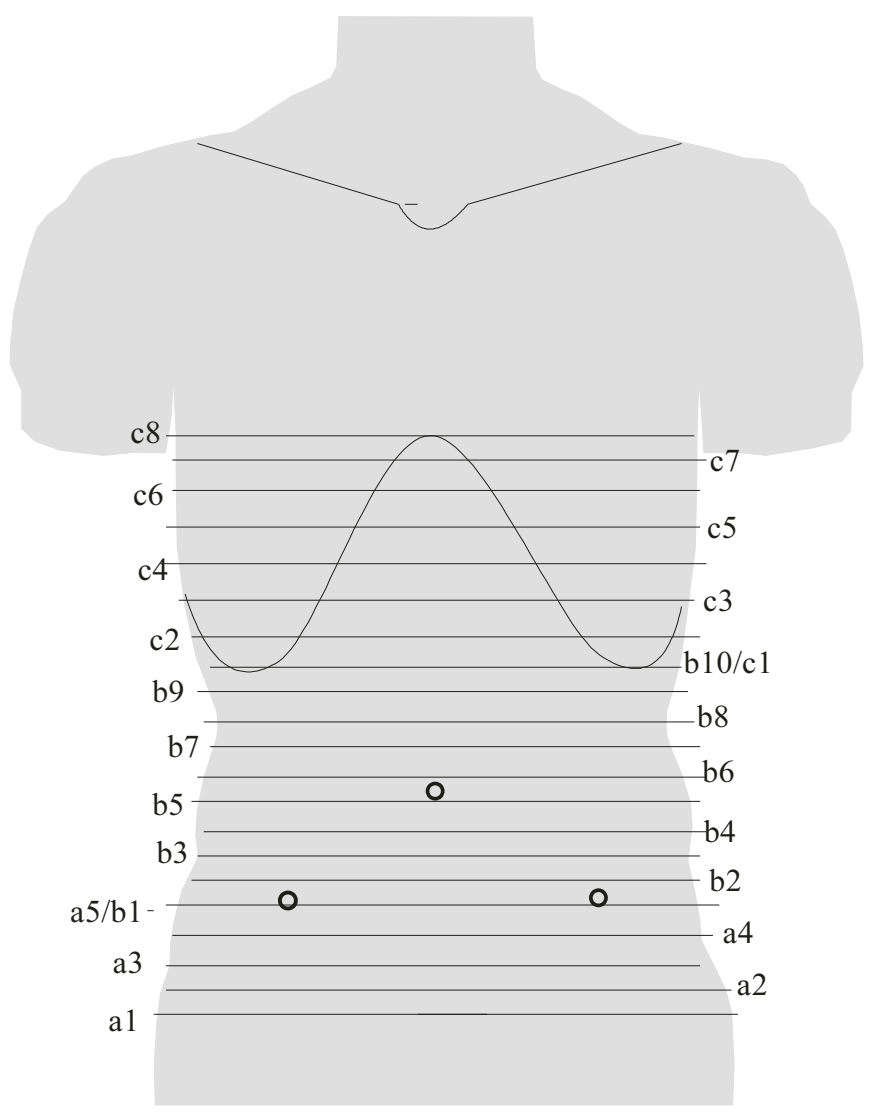

Abb. 1. Verwendetes Koordinatensystem.

Die Breite des M. rectus abdominis betrug hier 2,25 $\pm 0,39 \mathrm{~cm}$ rechts und 2,36 $\pm 0,42 \mathrm{~cm}$ links.

Zwischen Symphyse und Spinae iliacae anteriores superiores wurde der Mittelwert für die A. epigastrica inferior links mit $5,60 \pm 0,70 \mathrm{~cm}$ bei einem 95\%-Konfidenzintervall von $5,32-5,88 \mathrm{~cm}$ und rechts mit $5,65 \pm 0,73 \mathrm{~cm}$ bei einem $95 \%$ Konfidenzintervall von 5,36-5,94 cm gemessen.

Auf einer transversalen Linie zwischen den beiden Spinae iliacae anteriores superiores war die durchschnittliche Entfernung der A. epigastrica inferior von der Mittellinie links 4,56 $\pm 0,86 \mathrm{~cm}(95 \%$-Konfidenzintervall 4,23-4,89 cm) und rechts $4,65 \pm 0,95 \mathrm{~cm}$ (95\%-Konfidenzintervall 4,30-5,00 cm). Geschlechtsspezifische Unterschiede zeigten sich nicht. Die durchschnittliche Breite des Muskels betrug rechts 5,60 \pm 1,10 cm und links $5,73 \pm 0,86 \mathrm{~cm}$.

Auf Höhe des Nabels war die mittlere Entfernung der linken A. epigastrica inferior $4,28 \pm 0,70 \mathrm{~cm}$ bei einem $95 \%$-Konfidenzintervall von 4,00-4,56 cm. Auf der rechten Seite betrug diese Entfernung 4,08 \pm 0,74 cm bei einem 95\%-Konfidenzintervall von 3,80-4,36 cm. Die Mittelwerte männlicher Leichname lagen links $0,52 \mathrm{~cm}$ und rechts $0,21 \mathrm{~cm}$ über denen des weiblichen Kollektivs. Der Muskel wurde hier mit einer Breite von 7,10 $\pm 1,30 \mathrm{~cm}$ rechts und 7,35 $\pm 1,23 \mathrm{~cm}$ links gemessen. Bei 11 Präparaten ließen sich auf Höhe des unteren Rippenbogen linksseitig Gefäße darstellen, von denen 8 Anastomo- 


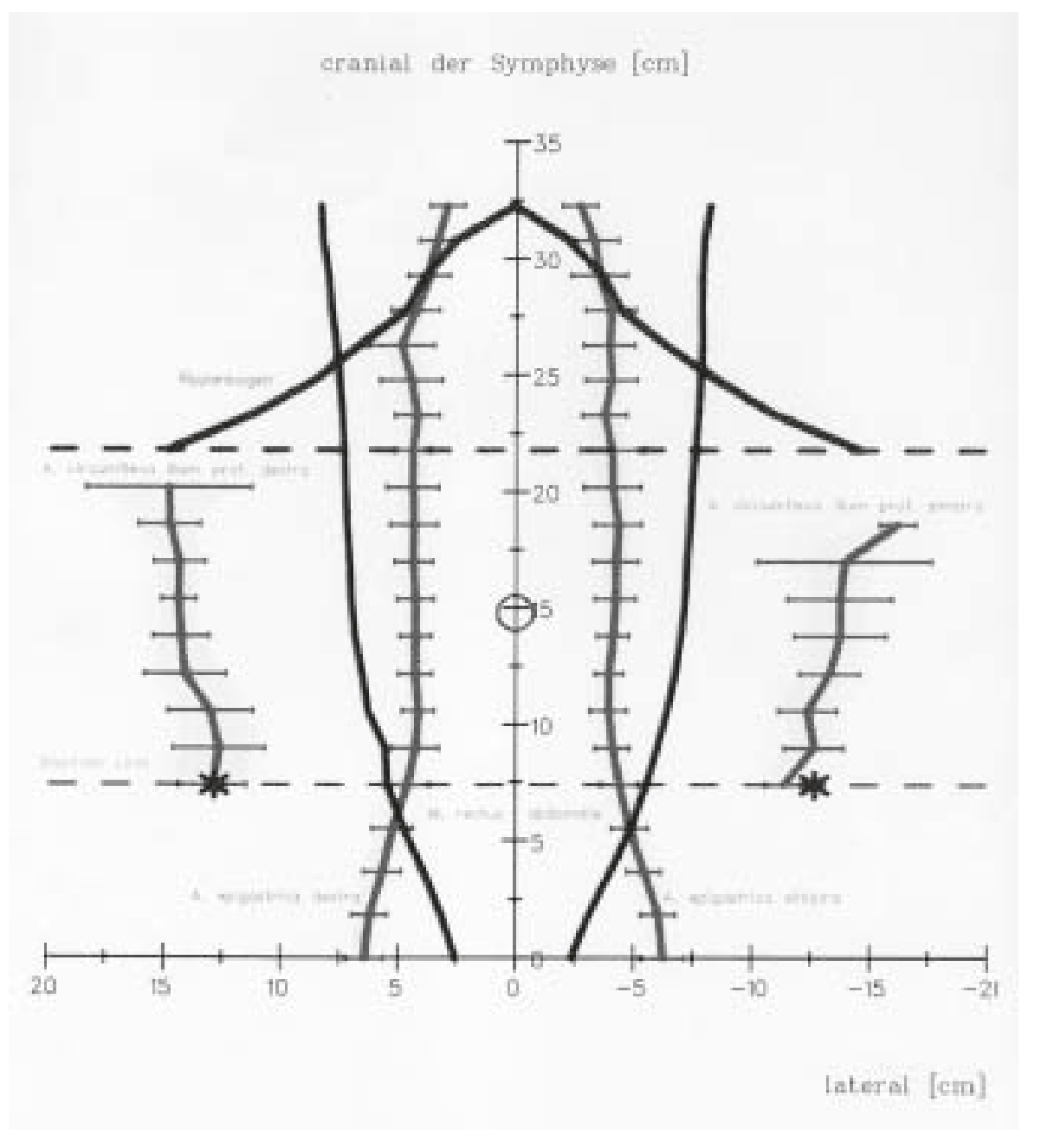

fenden Gefäße \pm Standardabweichung.

sen zwischen den Aa. epigasticae superior und inferior, 2 der A. epigastrica inferior und 1 der A. epigastrica superior entsprachen. Der Abstand zur Mitte betrug im Mittel 4,04 \pm 1,24 $\mathrm{cm}$ bei einem $95 \%$-Konfidenzintervall von 3,31-4,77 cm. Hier waren die Abstände bei männlichen Leichnamen im Mittel 2,02 cm größer als bei den weiblichen.

Auf Höhe des rechten unteren Rippenbogens konnten bei 13 Präparaten rechtsseitig Gefäße dargestellt werden, von denen 8 einer Anastomose zwischen A. epigastrica superior und inferior, 4 der A. epigastrica inferior und 1 der A. epigastrica superior entsprachen. Im Durchschnitt maß die Strecke zur Mittellinie 4,24 \pm 0,76 cm bei einem 95\%-Konfidenzintervall von 3,82-4,66 cm. Der Abstand der Präparate lag bei männlichen Leichnamen im Mittel um 0,48 cm über dem bei den weiblichen Leichnamen gemessenen. Der Abstand des lateralen Rands des M. rectus abdominis betrug hier rechts 7,60 \pm $1,51 \mathrm{~cm}$ und links $7,92 \pm 1,50 \mathrm{~cm}$.

Die A. epigastrica superior konnte am xypho-corposternalen Übergang links bei 17 Präparaten mit einem Mittelwert von $2,70 \pm 0,67 \mathrm{~cm}$ (95\%-Konfidenzintervall 2,38-3,02 cm) und rechts bei 18 Präparaten mit einem Mittelwert von 2,75 \pm 0,68 cm (95\%-Konfidenzintervall 2,43-3,07 cm) gemessen werden.
Links wurde der Unterschied von weiblichen zu männlichen Präparaten mit $0,23 \mathrm{~cm}$ gemessen, rechts betrug der Unterschied von männlichen zu weiblichen Leichnamen $0,39 \mathrm{~cm}$.

Der Verlauf des R. ascendens der A. circumflexa ilium profunda wurde zum lateralen Rand des M. rectus abdominis gemessen. Periumbilikal konnte dieses Gefäß bei 10 Präparaten links und bei 7 Präparaten rechts dargestellt werden. Im Mittel betrug die Distanz links 7,29 \pm 1,88 cm (95\%-Konfidenzintervall 6,13-8,45 cm) und rechts 7,44 $\pm 1,08 \mathrm{~cm}(95 \%$ Konfidenzintervall 6,64-8,24 cm). Unterhalb des rechten Rippenbogens (Meßpunkt b8) gelang die Darstellung dieses Gefäßes rechts nur in 3 Fällen (Mittelwert 7,53 $\pm 1,09 \mathrm{~cm}, 95 \%$ Konfidenzintervall 6,30-8,76 cm), links sogar nur in 2 Fällen (Mittelwert 8,85 \pm 0,55 cm, 95\%-Konfidenzintervall 8,09-9,61 $\mathrm{cm})$. Lediglich die Verläufe der rechten und linken A. epigastrica korrelierten an den meisten Messpunkten miteinander, eine eindeutige Korrelation zwischen dem Verlauf der A. epigastrica und dem M. rectus abdominis ergab sich nicht.

Den erarbeiteten Verlauf der Gefäße (mit der Standardabweichung bzw. dem 95\%-Konfidenzintervall) sowie des M. rectus abdominis einschließlich der anatomischen Markierungen zeigen die Abbildungen 2 und 3. 


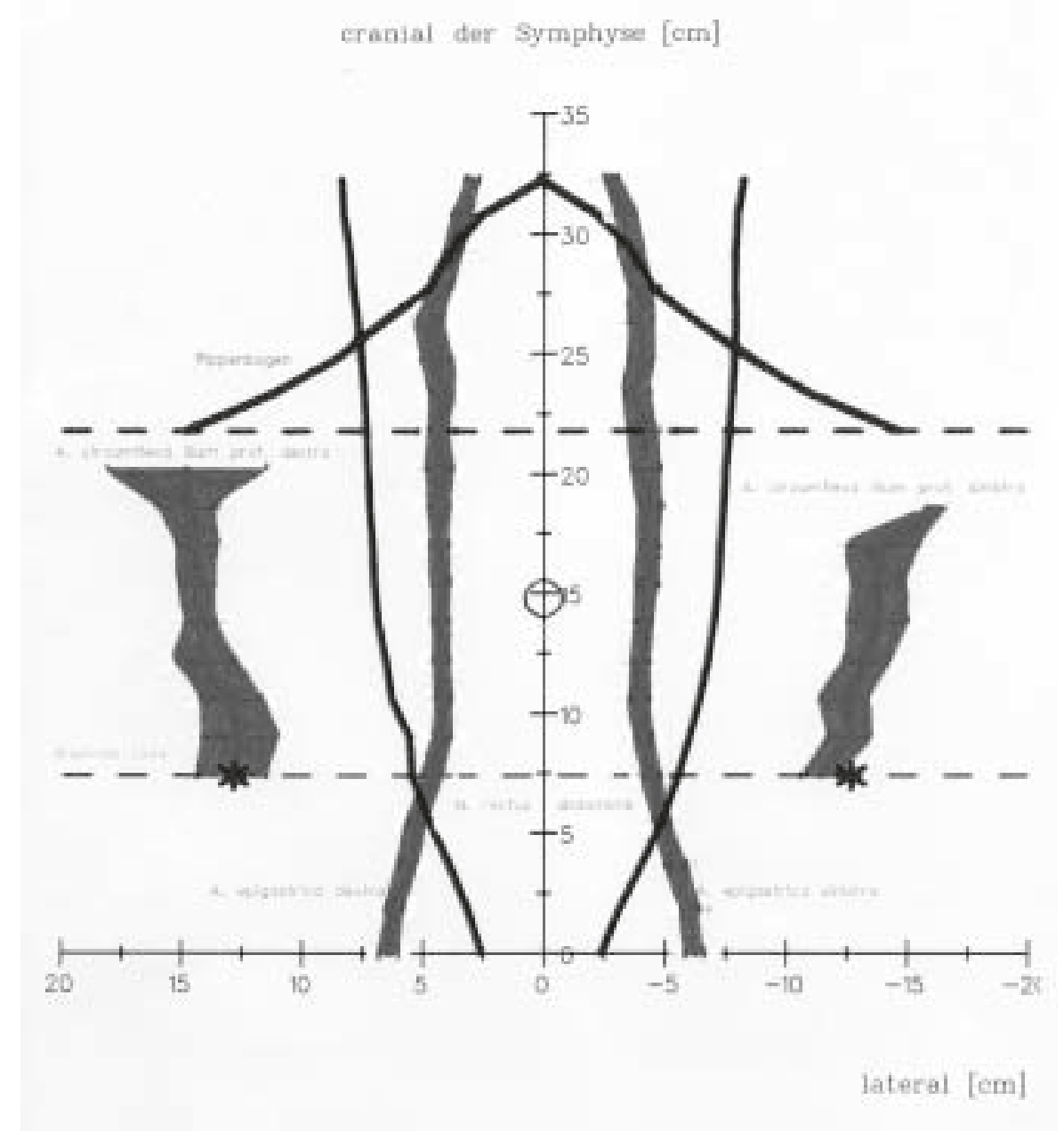

Abb. 3. Verlauf der Gefäße entsprechend dem errechneten $95 \%$-Konfidenzintervall.

\section{Diskussion}

Gefäßverletzungen der Bauchwand nach Trokarplatzierungen sind eine relevante Komplikation sämtlicher laparoskopischer Operationen, der in der Literatur nicht durchweg Beachtung geschenkt wird. Aus den obigen Daten ergeben sich idealtypische Verläufe der wichtigsten Bauchwandgefäße, wobei das Risiko einer Gefäßverletzung mit Zunahme des Abstands der Einstichstelle vom «Idealverlauf» exponentiell abnimmt.

Die in der eigenen Untersuchung ermittelten Ergebnisse stimmen mit denen von Hurd et al. [15] an 21 retrospektiv auf den Verlauf der Gefäße der Bauchwand untersuchten Computertomographien des Beckens bei Frauen überein. Darüber hinaus konnte der R. ascendens der A. circumflexa ilium profunda als ebenfalls verletzungsgefährdetes Gefäß identifiziert werden. Dieses Gefäß erscheint im Rahmen lateraler Trokarpositionen bei der Cholezystektomie, Kolonchirurgie, Refluxchirurgie und Hernienreparation gefährdet.

Folgende «dangerous zones» können festgelegt werden: zwischen Symphyse und Spinae in einem Streifen 3,5-8 cm lateral der Mittellinie, zwischen den Spinae iliacae anteriores superiores und dem Nabel $2 \mathrm{~cm}$ lateral der Mittellinie bis zum late- ralen Rand des M. rectus sowie 5-9 cm lateral des M. rectus abdominis (Abb. 4). Im Epigastrium (zwischen dem unteren und oberen Rippenbogenrand) sind Trokareinstiche perimedian sowie ab $5 \mathrm{~cm}$ lateral der Mittellinie sicher. Bedingt durch die sich widerspiegelnde Variabilität des anatomischen Verlaufs der Bauchwandgefäße können nur Empfehlungen für die Position von Trokaren anhand von Anhaltswerten gegeben werden. Legt man wie hier das $95 \%$-Konfidenzintervall zugrunde, so ergeben sich damit «dangerous zones» für die Trokarplatzierung. Diese Abstände werden sich in der chirurgischen Wirklichkeit nicht immer einhalten lassen. Die Kenntnis der vaskulären Anatomie der Bauchwand ist jedoch nichtsdestoweniger die Voraussetzung, Gefäßverletzungen durch Trokare zu vermeiden. 


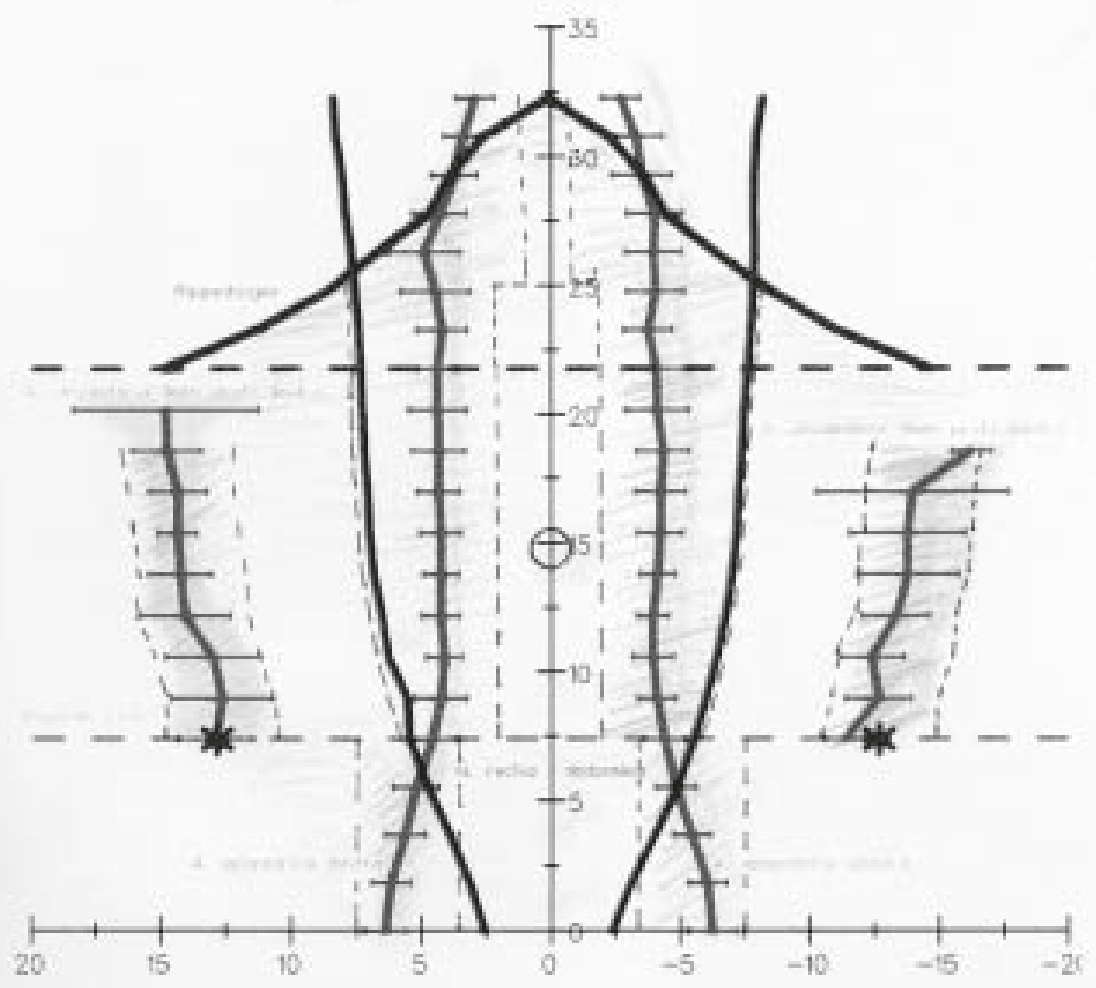

Abb. 4. Darstellung gefährlicher (schraffiert) und ungefährlicher Regionen zur Trokarplatzierung.

\section{Literatur}

1 Hurd WW, Pearl ML, DeLancey, JOL, Quint EH, Garnett B, Bude RO: Laparoscopic injury of abdominal wall blood vessels: A report of three cases. Obstet Gynecol 1993;82:673-676.

2 Nordestgaard AG, Bodily KC, Osborne RW, Buttorff JD: Major vascular injuries during laparoscopic procedures. Am J Surg 1995;169:543-545.

3 Pring DW: Inferior epigastric hemorrhage, an avoidable complication of laparoscopic clip sterilization. Br J Obstet Gynaecol 1983;90:480-482.

4 Soderstrom RM: Injuries to major blood vessels during endoscopy. J Am Assoc Gynecol Laparosc 1997;4:395-398.

5 Milloy FJ, Anson BJ, McAffee DK: The rectus abdominis muscle and the epigastric arteries. Surg Gynecol Obstet 1960;110:293-302.

6 Von Waldeyer AJ, Fanghänel J, Pera F, Anderhuber F, Nitsch R (Hrsg): Anatomie des Menschen. Berlin, de Gruyter, 2003, p 656.
7 Kane MG, Krejs GJ: Complications of diagnostic laparoscopy in Dallas: A 7-year prospective study. Gastrointest Endosc 1984;30:237-240.

8 Loffer FD, Pent D: Indications, contraindications and complications of laparoscopy. Surg Gynecol Surv 1975;30:407-427.

9 Mintz M: Risks and prophylaxis in laparoscopy: A survey of 100000 cases. J Reprod Med 1977;18: 269-272.

10 Leibl BJ, Schmedt CG, Schwarz J, Kraft K, Bittner $\mathrm{R}$ : Laparoscopic surgery complications associated with trocar tip design: Review of literature and own results. J Laparoendosc Adv Surg Tech A 1999;9: 135-140.

11 Petrossian E, Menegus MA, Issenberg HJ, Jones A, Frame R, Brodman RF: Ultrasound evaluation of the inferior epigastric artery. Ann Thorac Surg 1994;57:895-898.
12 Frangenheim H: Sicherheitsmaßnahmen zur Verhütung von Komplikationen bei der Laparoskopie. Endoscopy 1971;1:10-20.

13 Quint EH, Wang FL, Hurd WH: Laparoscopic transillumination for the location of anterior abdominal wall vessels. J Laparoendosc Surg 1996;6: 167-169.

14 Hurd WW, Wang L, Schemmel MT: A comparison of the relative risk of vessel injury with conical versus pyramidal laparoscopic trocars in a rabbit model. Am J Obste. Gynecol 1995;173:1731-1733.

15 Hurd WW, Bude RO, DeLancey JOL, Newman JS: The location of abdominal wall blood vessels in relationship to abdominal landmarks apparent at laparoscopy. Am J Obstet Gynecol 1994;171:642-646. 